\title{
Observations from stress testing in the troponin twilight zone
}

\author{
Michael C. Kontos, $M D,{ }^{a}$ and Todd C. Villines, $M D^{b}$ \\ a Division of Cardiology, Department of Internal Medicine, Pauley Heart Center, Virginia \\ Commonwealth University, Richmond, VA \\ b Department of Internal Medicine, University of Virginia Health System, Charlottesville, VA
}

Received Mar 3, 2020; accepted Mar 4, 2020

doi: $10.1007 / \mathrm{s} 12350-020-02147-8$

\section{See related article, pp. $2941-2948$}

Over the past 30 years, there has been an incremental increase in the sensitivity of troponin (Tn) assays, with each new Tn assay generation able to detect smaller amounts of myocardial necrosis. High sensitivity troponin (hsTn) assays, which have just recently become available in the United States (US), are those that are able to detect Tn in at least $50 \%$ of "normal", patients, with abnormal values typically defined as those above the 99th percentile. ${ }^{1}$ Although the first hsTn assay was introduced in Europe about 10 years ago, the first assay (hsTnT) was only approved for use in the US in 2017; currently four hsTn assays are now available (1 hsTnT, 3 hsTnI).

Routine use of hsTn has important implications for the care of patients presenting to the Emergency Department (ED), leading to early and accurate risk stratification of patients with possible myocardial infarction (MI). Detecting low but abnormal Tn values allows earlier diagnosis, potentially leading to earlier triage, treatment, and admission. Conversely, undetectable to very low and stable hsTn levels allows rapid MI exclusion, leading to earlier discharge. Numerous strategies assessing hsTn for ED MI diagnosis have been evaluated. ${ }^{2-8}$ One protocol that uses hsTnT, currently recommended by the European Society of Cardiology

\footnotetext{
Reprint requests: Michael C. Kontos, MD, Division of Cardiology, Department of Internal Medicine, Pauley Heart Center, Virginia Commonwealth University, Room 285 Gateway Building, 2nd floor Gateway, 1200 E Marshall St, PO Box 980051Richmond, VA 23298-0051; Michael.kontos@vcuhealth.org

J Nucl Cardiol 2021;28:2949-51.

$1071-3581 / \$ 34.00$

Copyright (C) 2020 American Society of Nuclear Cardiology.
}

and used in the current study, ${ }^{2,6-8}$ classifies patients into three groups based on their initial and follow-up hsTn one hour later. Patients classified as low risk are eligible for early discharge if they have undetectable or very low hsTnT levels at the time of presentation, or there is a minimal change on repeat testing 1 hour later. Patients who have significantly elevated hsTnT are classified as abnormal $^{8}$ or as having MI, are typically admitted for further evaluation. Patients who do not fall into either of these two groups are classified as "observation" and undergo further evaluation. It is this group of patients that the current study focuses on.

In the current study, ${ }^{9}$ the authors report on a retrospective cohort of consecutive patients presenting to the ED with possible ACS at their institution for rest stress MPI (either SPECT or PET) who had mildly abnormal hsTnT values who met criteria for the observation zone. Patients were additionally classified based on the HEART score. ${ }^{10}$ Among the 213 patients included from the total of 1705 patients with intermediate hsTnT during the 7-month study period, risk factors for coronary disease were common (86\% had hypertension, 60\% increased cholesterol and $40 \%$ had diabetes), and the majority (53\%) of patients had known coronary artery disease (CAD). Myocardial ischemia, defined as a reversible perfusion defect with a positive summed difference score, was present in 29 (13.6\%) patients with $21(9.9 \%)$ patients having a summed difference score of $\geq 4$; only $16(7.5 \%)$ had a myocardial risk area of $>10 \%$. The strongest predictor of any ischemia, an outcome defined in a binary fashion, was the presence of known CAD. Specifically, 27 of 29 patients with an abnormal stress MPI had a known history of CAD compared to only 2 patients without known CAD. The majority of patients with ischemia on stress MPI did not ultimately undergo catheterization or revascularization.

The authors should be congratulated on their important study, which represents one of the few to date and the first in the US cohort to report on stress 
myocardial perfusion testing results in this important group of patients. As such, it underlines the significant limitations in the data currently available and serves as a call to action for more research to help define optimal care pathways for this important patient cohort. Most prior studies reporting on the utility of hsTn for ED use have limited information on the types of diagnostic testing performed, ${ }^{2,4,7,8}$ as well as lack detailed information on test characteristics on those who do undergo additional testing. ${ }^{5,6}$ In one of the few available studies, Nestelberger et al reported outcomes in patients referred for observation. ${ }^{11}$ Similar to the current study, a small minority underwent stress MPI, of whom approximately half had evidence of ischemia. In contrast to the current study, about half the patients in the observation zone underwent invasive coronary angiography. ${ }^{11}$

In the current study, the authors note that higher HEART scores were associated with myocardial ischemia. This is expected as three of the components of the score ( $\mathrm{H}$-history, A-age, $\mathrm{R}$-risk factors/known CAD) also predict CAD. The converse was also true and may be the more important finding; no patients who had intermediate hsTn levels with a HEART scores of 1 or 2 had ischemia, while only 3 patients with a HEART score of 3 had myocardial ischemia. If this is a consistent finding across the cohort of intermediate hsTn results, then it would appear that these patients are low risk and would be safe for either no stress testing or stress testing performed on an outpatient basis rather than admission.

Finally, the authors found that testing was safe. This is reassuring but not unexpected. Pharmacologic stress MPI in patients after MI is usually low risk, even if performed as early as 1 day after MI. ${ }^{12}$ Most patients in the current study did not undergo early testing, defined as on the day of presentation, with unclear numbers having exercise vs pharmacologic testing.

Some aspects of the current study deserve additional consideration. The number of patients included represented only $12.5 \%$ of all patients with observation zone hsTnT values and only those referred to stress MPI, making this a highly selected population. Testing patterns and clinical outcomes of patients with similar hsTn values who were not referred for stress MPI were not explored and are of significant interest. As stated by the authors, very few patients were referred for other noninvasive testing modalities (e.g., stress echocardiogram or coronary CT angiography). Despite the low rates of testing, clinical outcomes were uncommon during the 90 days after discharge.

It is important to note that the manufacturer's recommended 99th percentile for this assay is $14 \mathrm{ng} / \mathrm{L}$; therefore, many of the patients in the observation group would meet criteria for chronic, rather than acute myocardial injury. The differentiation is important.
Chronic Tn elevations reflect ongoing myocardial injury, and often indicates some type of structural heart disease, such as CAD, hypertrophy, systolic dysfunction or significant valvular abnormalities. Although often considered a nuisance diagnosis, as reflected in terminology such as "troponinosis" or "troponin leak", 13 the presence of these increased Tn levels identifies a cohort of patients at substantially increased risk of mortality. Although not at immediate risk for cardiac events, these patients have a significantly higher cardiovascular event rate over long-term follow up, with a mortality at least twice that of type 1 MI. ${ }^{14,15}$ Detection of these low level hsTnT values should not be considered a benign event, rather they should be the impetus for more aggressive secondary risk factor modification.

Because these patients underwent ED evaluation for possible MI, it is likely that many of the patients included presented with symptoms consistent with myocardial ischemia. The decision to perform additional testing to assess for ACS and the choice of test will be dependent on specific institutional and patient characteristics. In several prospective randomized controlled studies of lower risk ED patients, CT coronary angiography has provided rapid, accurate, and safe diagnostic evaluation. However, the use of coronary CT angiography is best suited for patients without known CAD and would not have been ideal for a large proportion of patients (53\%) in this study. Additionally, the presence of renal failure or other contraindications may preclude its routine use ${ }^{11}$ suggesting that CTA may not be an option for the majority of observation zone patients.

It is somewhat surprising that more patients who underwent MPI did not have myocardial ischemia, given the association of elevated hsTn with underlying coronary disease, ${ }^{16,17}$ and the possibility some of these patients were late presenting MI patients. ${ }^{18}$ This may relate to selection bias inherent in retrospective, singlecenter studies involving those clinically referred for a single testing modality. For example, patients with more concerning clinical symptoms for ACS may have been referred for invasive coronary angiography and those with less compelling symptoms or available prior testing results treated medically or evaluated as an outpatient.

It would have been helpful if the authors had provided the total number of patients undergoing ACS risk assessment. Prior studies have found that approximately 20 to $30 \%$ of patients fall in this range. ${ }^{2,4,6,7,11}$ Whether the authors' data are similar is unknown and would have provided some insights on the US population. Additionally, it is unclear how many patients had PET vs SPECT imaging, or how many underwent exercise vs pharmacologic testing. PET imaging may allow for increased sensitivity and overall accuracy as compared 
to SPECT imaging and its performance in this cohort is of significant clinical interest.

One concern shared by many US providers is that the more frequent detection of hsTn will result in higher rates of additional cardiovascular testing, particularly given the lower threshold for Tn testing in the US. ${ }^{19}$ In non-US cohorts, this has fortunately not been the case. $^{3,5,6}$ The current study appears consistent with these results, given the small proportion of patients in the observation zone who ultimately underwent additional cardiovascular testing in the inpatient setting.

In summary, the current study represents an important single-center observational experience involving patients who account for 20 to $30 \%$ of ED patients with possible ACS using contemporary hsTn assays. $^{2,4,6,7,11,20}$ As the authors pointed out, additional information on what test to perform, if any, as well as the timing of testing (inpatient or outpatient), is needed, particularly as the use of hsTn assays are incorporated in the standard ED MI assessment in the United States over the next few years.

\section{References}

1. Wu AHB, Christenson RH, Greene DN, Jaffe AS, Kavsak PA, Ordonez-Llanos J, et al. Clinical Laboratory Practice Recommendations for the Use of Cardiac Troponin in Acute Coronary Syndrome: Expert Opinion from the Academy of the American Association for Clinical Chemistry and the Task Force on Clinical Applications of Cardiac Bio-Markers of the International Federation of Clinical Chemistry and Laboratory Medicine. Clin Chem 2018;64:645-55.

2. Mueller C, Giannitsis E, Christ M, Ordóñez-Llanos J, deFilippi C, McCord J, et al. Multicenter Evaluation of a 0-Hour/1-Hour Algorithm in the Diagnosis of Myocardial Infarction With HighSensitivity Cardiac Troponin T. Ann Emerg Med 2016;68:76-87.

3. Shah AS, Anand A, Sandoval Y, Lee KK, Smith SW, Adamson $\mathrm{PD}$, et al. High-sensitivity cardiac troponin I at presentation in patients with suspected acute coronary syndrome: a cohort study. Lancet 2015;386:2481-8.

4. Nowak RM, Christenson RH, Jacobsen G, McCord J, Apple FS, Singer AJ, et al. Performance of novel high-sensitivity cardiac troponin $\mathrm{i}$ assays for $0 / 1$-hour and $0 / 2$ - to 3-hour evaluations for acute myocardial infarction: results from the HIGH-US study. Ann Emerg Med 2020, in press.

5. Chew DP, Lambrakis K, Blyth A, Seshadri A, Edmonds MJR, Briffa T, et al. A randomized trial of a 1-hour troponin t protocol in suspected acute coronary syndromes: the rapid assessment of possible acute coronary syndrome in the emergency department with high-sensitivity troponin T study (RAPID-TnT). Circulation 2019;140:1543-56.

6. Twerenbold R, Costabel JP, Nestelberger T, Campos R, Wussler D, Arbucci R, et al. Outcome of applying the esc 0/1-hour algorithm in patients with suspected myocardial infarction. J Am Coll Cardiol. 2019;74:483-94.

7. Stoyanov KM, Hund H, Biener M, Gandowitz J, Riedle C, Löhr J, et al. RAPID-CPU: a prospective study on implementation of the ESC 0/1-hour algorithm and safety of discharge after rule-out of myocardial infarction. Eur Heart J Acute Cardiovasc Care 2019, in press.

8. Vigen R, Kutscher P, Fernandez F, Yu A, Bertulfo B, Hashim IA, et al. Evaluation of a novel rule-out myocardial infarction protocol incorporating high-sensitivity troponin $\mathrm{t}$ in a us hospital. Circulation 2018;138:2061-3.

9. Khan A, Engineer R, Wang S, Jaber WA, Menon V, Cremer PC. Initial experience regarding the safety and yield of rest-stress myocardial perfusion imaging in emergency department patients with mildly abnormal high-sensitivity cardiac troponins $\mathrm{J}$ Nucl Cardiol 2020

10. Six AJ, Backus BE, Kelder JC. Chest pain in the emergency room: value of the HEART score. Neth Heart J 2008;16:191-6.

11. Nestelberger T, Wildi K, Boeddinghaus J, Twerenbold R, Reichlin T, Giménez MR, et al. Characterization of the observe zone of the ESC 2015 high-sensitivity cardiac troponin 0h/1h-algorithm for the early diagnosis of acute myocardial infarction. Int $\mathbf{J}$ Cardiol 2016;207:238-45

12. Mahmarian JJ, Shaw LJ, Filipchuk NG, Dakik HA, Iskander SS, Ruddy TD, et al. A multinational study to establish the value of early adenosine technetium-99m sestamibi myocardial perfusion imaging in identifying a low-risk group for early hospital discharge after acute myocardial infarction. J Am Coll Cardiol 2006;19(48):2448-57.

13. Januzzi JL Jr, Mahler SA, Christenson RH, Rymer J, Newby LK, Body R, et al. Recommendations for institutions transitioning to high-sensitivity troponin testing: JACC scientific expert panel. J Am Coll Cardiol 2019;73:1059-77.

14. Sarkisian L, Saaby L, Poulsen TS, Gerke O, Jangaard N, Hosbond $\mathrm{S}$, et al. Clinical characteristics and outcomes of patients with myocardial infarction, myocardial injury, and nonelevated troponins. Am J Med 2016;129(446):e5-446.

15. Cediel G, Gonzalez-Del-Hoyo M, Carrasquer A, Sanchez R, Boqué C, Bardají A. Outcomes with type 2 myocardial infarction compared with non-ischaemic myocardial injury. Heart 2017;103:616-22.

16. Adamson PD, Hunter A, Madsen DM, Shah ASV, McAllister DA, Pawade TA, et al. High-sensitivity cardiac troponin I and the diagnosis of coronary artery disease in patients with suspected angina pectoris. Circ Cardiovasc Qual Outcomes 2018;11:e004227.

17. Januzzi JL Jr, Suchindran S, Coles A, Ferencik M, Patel MR, Hoffmann U, et al. High-sensitivity troponin I and coronary computed tomography in symptomatic outpatients with suspected CAD: insights from the PROMISE trial. JACC Cardiovasc Imaging 2019;12:1047-55.

18. Bjurman C, Larsson M, Johanson P, Petzold M, Lindahl B, Fu MLX, et al. Small changes in troponin $\mathrm{T}$ levels are common in patients with non-ST-segment elevation myocardial infarction and are linked to higher mortality. J Am Coll Cardiol 2011;62:1231-8.

19. Shah ASV, Sandoval Y, Noaman A, Sexter A, Vaswani A, Smith SW, et al. Patient selection for high sensitivity cardiac troponin testing and diagnosis of myocardial infarction: prospective cohort study. BMJ 2017;359:j4788.

20. Twerenbold R, Jaeger C, Rubini Gimenez M, Wildi K, Reichlin T, Nestelberger $\mathrm{T}$, et al. Impact of high-sensitivity cardiac troponin on use of coronary angiography, cardiac stress testing, and time to discharge in suspected acute myocardial infarction. Eur Heart $\mathbf{J}$ 2016;37:3324-32.

Publisher's Note Springer Nature remains neutral with regard to jurisdictional claims in published maps and institutional affiliations. 